\title{
GAC via Unit Propagation
}

\author{
Fahiem Bacchus \\ Department of Computer Science,University of Toronto, Canada. \\ fbacchus@cs.toronto.edu
}

\begin{abstract}
In this paper we argue that an attractive and potentially very general way of achieving generalized arc consistency (GAC) on a constraint is by using unit propagation (UP) over a CNF encoding of the constraint. This approach to GAC offers a number of advantages over traditional constraint specific algorithms (propagators): it is easier to implement, it automatically provides incrementality and decrementality in a backtracking context, and it can provide clausal reasons to support learning and non-chronological backtracking. Although UP on standard CNF encodings of a constraint fails to achieve GAC, we show here that alternate CNF encodings can be used on which UP does achieve GAC. We provide a generic encoding applicable to any constraint. We also give structure specific encodings for the regular, among, and gen-sequence constraints on which UP can achieve GAC with the same run time bounds as previously presented propagators. Finally, we explain how a UP engine can be added to a CSP solver to achieve a seamless integration of constraints encoded in CNF and propagated via UP and those propagated via traditional constraint specific propagators.
\end{abstract}

\section{Introduction}

Unit propagation (UP) is a local propagation mechanism for propositional formulas expressed in conjunctive normal form (CNF). Any constraint over finite domain variables can be converted to CNF using the direct encoding of [17]. However, UP on that encoding (or on the other encodings presented in [17]) does not achieve GAC - it has only the power of forward checking. In this paper we demonstrate that alternate CNF encodings can be constructed that allow UP to efficiently achieve GAC.

Using UP on a CNF encoding to achieve GAC has a number of advantages over the specialized constraint specific algorithms (propagators) that are typically used. Intuitively, these advantages arise from the fact that propagators are procedural where as the $\mathrm{CNF}$ encoding utilized by UP is declarative. In particular, the sequencing of operations defining a propagator is typically quite rigid. Altering these operations so as to support features like interleaving with the propagators for other constraints or incrementality and decrementality in a backtracking context often requires non-trivial modifications.

In contrast UP can be run on a CNF encoding in flexible ways. In particular, UP can easily interleave the propagation of multiple constraints; it is always incremental; decrementality can be achieved almost without cost; and it readily supports the derivation of new clauses (generalized nogoods [12]) that can be used to improve search performance via learning and non-chronological backtracking. First, once encoded in CNF a constraint's clauses need not be distinguished from the clauses encoding other 
constraints. Thus, UP can interleave its GAC propagation across different constraints in arbitrary ways. For example, if the constraints $C_{1}$ and $C_{2}$ are both encoded in CNF then UP can detect that a value for a variable has become inconsistent for constraint $C_{1}$ while it is in the middle of propagating the clauses for computing GAC on $C_{2}$. Furthermore, this pruned value will then automatically be taken into account during the rest of UP's computation of GAC on $C_{2}$. Second, UP works by forcing the truth value of propositions; the order in which these truth values are set is irrelevant to the final result. Hence, it is irrelevant to UP if some of these propositions already have their truth value set: i.e., UP is always incremental. Third, the modern technique for implementing UP employs a lazy data structure called watch literals. One of the salient features of watch literals is that they need not be updated on backtrack. Thus, with UP decrementality can be achieved essentially without cost: only the forced truth assignments need to be undone. Fourth, if UP detects a contradiction, existent techniques can be used to learn powerful nogoods that can improve search performance. These techniques learn generalized nogoods (clauses) which are more powerful than standard nogoods [12].

Previous work has investigated the connections between SAT propagation mechanisms (including UP) and various CSP propagation mechanisms (e.g., [6, 3, 9, 17]). Most of this work, however, has been confined to binary constraints and thus has addressed AC rather than GAC. However, Hebrard et al. [11] building on the work of [9] present an CNF encoding for an arbitrary constraint that allows UP to achieve relational $\mathrm{k}$-arc-consistency. Although that paper did not directly address achieving GAC via UP, their encoding can easily be adapted accomplish this. Furthermore, UP on this encoding achieves GAC in the same time complexity as a generic GAC algorithm like GAC-Schema [4] (GAC-Schema can however have a lower space complexity). Here one of our contributions is to elaborate the connection between GAC and UP on generic constraints, showing how Hebrard et al.'s encoding idea can be adapted to achieve GAC and how a more direct CNF encoding of the constraint can also allow UP to achieve GAC.

The main benefit of GAC in Constraint Programming, however, lies not so much with generic constraints, but rather in the fact that for a number of constraints specialized algorithms exist, called propagators, that can compute GAC in time that is typically polynomial in the arity of the constraint. This is a significant speed up over generic GAC algorithms which require time exponential in the arity of the constraint.

The main contribution of this paper is to demonstrate that just as some constraints admit specialized algorithms for computing GAC, some constraints also admit specialized CNF encodings on which UP can compute GAC much more efficiently. In this paper, we demonstrate such encodings for three different constraints: regular, among and gen-sequence. However, UP on our encoding for gen-sequence is not quite sufficient to achieve GAC. Nevertheless, a simple extension of UP called the failed literal test is able to achieve GAC on this encoding. The failed literal test retains the advantages of UP mentioned above.

An additional contribution of the paper is to explain how a CSP solver can easily be modified to integrate a state of the art UP engine that can then be used to achieve $\mathrm{GAC}$ for some constraints while the other constraints are propagated using traditional mechanisms. It is worth noting that a UP engine does not need to be implemented 
from scratch. Rather, very efficient state of the art UP engines are publically available. For example, the open source MiniSat SAT solver [7] (a consistent winner of recent SAT solver competitions) contains a clearly coded UP engine that can be extracted and utilized for this purpose- the code can be freely used even for commercial purposes.

In the sequel we first present the background properties of GAC and UP needed for the rest of the paper, and explain what is already known about the connection between GAC and UP. We then give a new result that elaborates on this connection. These results illustrate how generic GAC can be accomplished by UP. We then illustrate how a UP engine can be seamlessly integrated into a CSP solver. Turning to global constraints, we then present encodings for the afore mentioned constraints that allow UP to achieve GAC in polynomial time. We close with some final conclusions and ideas for future work.

\section{Background}

Let $\mathcal{V}=\left\{V_{1}, \ldots, V_{n}\right\}$ be a sequence of variables each of which has a finite domain of possible values $\operatorname{dom}\left[V_{i}\right]$. Corresponding to the variable sequence $\mathcal{V}$ is the Cartesian product of the variable domains. We extend $d o m$ to apply to sequences of variables: $\operatorname{dom}[\mathcal{V}]=\operatorname{dom}\left[V_{1}\right] \times \cdots \times \operatorname{dom}\left[V_{n}\right]$. A constraint $C$ is a function over a sequence of variables scope $(C)$. The size of scope $(C)$ is called the arity of $C . C$ maps tuples from $\operatorname{dom}[\operatorname{scope}(C)]$ to true/false. That is, if $\operatorname{scope}(C)=\left\langle X_{1}, \ldots, X_{k}\right\rangle$ and $\tau \in$ $\operatorname{dom}\left[X_{1}\right] \times \cdots \times \operatorname{dom}\left[X_{k}\right]$, then $C(\tau)=$ true/false. If $C(\tau)=$ true we say that $\tau$ satisfies $C$, else it falsifies it. We also view $\tau$ as being a set of assignments and say that $V=d \in \tau$ if $\tau$ 's $V$ 'th dimension is equal to $d$.

A constraint $C$ is said to be GAC if for every variable $X \in \operatorname{scope}(C)$ and every value $d \in \operatorname{dom}[X]$ there exists a $\tau \in \operatorname{dom}[\operatorname{scope}(C)]$ with $X=d \in \tau$ and $C(\tau)=$ true. A satisfying tuple $\tau$ containing $X=d$ is called a support for $X=d$. A constraint can be made GAC by simply removing every unsupported value from the domains of its variables. If the value $d \in \operatorname{dom}[V]$ is unsupported by $C$ we say that the assignment $V=d$ is GAC-inconsistent for $C$.

GAC propagation is the process of making some or all of the constraints of the problem GAC. If more than one constraint is to be made GAC, then GAC propagation will be an iterative process as making one constraint GAC might cause another constraint to no longer be GAC. However, since enforcing GAC (making a constraint GAC) is a monotonic process of removing unsupported values from the domains of variables, propagation must converge in at most a polynomial number of steps.

Unit propagation (UP) works on propositional formulas expressed in conjunctive normal form (CNF). A CNF formula is a conjunction of clauses, each of which is a disjunction of literals, each of which is a variable of the formula valued either positively or negatively. Given a CNF formula $F$ and a literal $\ell$ we denote the reduction of $F$ by $\ell$ as $\left.F\right|_{\ell}$. The reduction $\left.F\right|_{\ell}$ is a new CNF formula obtained from $F$ by removing all clauses of $F$ containing $\ell$ and then removing $\neg \ell$ (the negation of $\ell$ ) from all remaining clauses. UP works by iteratively identifying all unit clauses of $F$ (i.e., clauses containing only a single literal). Each unit clause $(\ell) \in F$ entails that $\ell$ must be true. Hence if $(\ell)$ is a unit clause of $F$ then $F$ is equivalent to $\left.F\right|_{\ell}$. UP forces $\ell$ and transforms $F$ to $\left.F\right|_{\ell}$ 
whenever it finds a unit clause $(\ell)$. Furthermore, since $\left.F\right|_{\ell}$ might contain additional unit clauses UP iteratively identifies unit clauses and continues to reduce the formula until no more units exist.

It is not hard to demonstrate that the final formula produced by UP does not depend on the order in which UP removes the unit clauses. Similarly, if GAC is being achieved for a collection of constraints, the order in which these constraints are processed (and reprocessed) has no effect on the final set of reduced variable domains. It can also be seen that UP runs in time linear in the total size of the input formula $F$ (the sum of the lengths of the clauses of $F$ ): each clause of length $k$ need only be visited at most $k$ times to remove literals or force the last literal during UP. GAC also runs in time linear in the size of a tabular representation of the constraint: by processing each satisfying tuple of the constraint GAC can identify all supported variable values. ${ }^{1}$ However, the tabular representation of a constraint has size exponential in the constraint's arity. It can be shown that when taking the arity of the constraint as the complexity parameter achieving GAC is NP-Hard. Both UP and GAC are sound rules of inference. That is, if $d$ is removed from the domain of $V$ by GAC, then no tuple of assignments satisfying $C$ can contain $V=d$. Similarly, if UP forces the literal $\ell$ then $\ell$ must be true in all truth assignments satisfying $F$. Finally, Both GAC and UP can detect contradictions. If UP produces an empty clause during its propagation, the initial formula is UNSAT, and if GAC produces an empty variable domain the constraint cannot be satisfied.

UP can also be utilized for look-ahead. In particular the failed literal rule [8] involves adding the unit clause $(\ell)$ and then doing UP. If this yields a contradiction we can conclude that $\neg \ell$ is implied by the original formula.

\subsection{Achieving generic GAC with UP}

In [11] a CNF encoding for a constraint was given on which UP is able to achieve relational $k$-arc consistency. Although GAC was not mentioned in that paper, their idea can be easily be adapted to allow UP to compute GAC on an arbitrary constraint.

The required encoding contains clauses for representing the variables and their domains of possible values. We call these clauses the Variable Domain Encoding (VDom). VDom consists of the following propositional variables and clauses. For every multi-valued variable $V$ with $\operatorname{dom}[V]=\left\{d_{1}, \ldots, d_{m}\right\}$ :

1. $m$ assignment variables $A_{V=d_{j}}$ one for each value $d_{j} \in d o m[V]$. This variable is true when $V=d_{j}$, it is false when $V$ cannot be assigned the value $d_{j}$, i.e., $V$ has been assigned another value, or $d_{j}$ has been pruned from $V$ 's domain.

2. The $O\left(m^{2}\right)$ binary clauses $\left(\neg A_{V=d}, \neg A_{V=d^{\prime}}\right)$ for every $d, d^{\prime} \in \operatorname{dom}[V]$ such that $d \neq d^{\prime}$, capturing the condition that $V$ cannot be assigned two different values.

3. The single clause $\left(A_{V=d_{1}}, \ldots, A_{V=d_{m}}\right)$ which captures the condition that $V$ must be assigned some value.

One important point about the VDom clauses is that although there are $O\left(\mathrm{~km}^{2}\right)$ clauses, UP can be performed on these clauses in time $O(\mathrm{~km})$. In particular, it is easy

\footnotetext{
${ }^{1}$ Practical GAC algorithms employ support data structures that make re-establishing GAC more efficient and that save space when the constraint is intensionally represented.
} 
to modify a UP engine so that the information contained in the VDom clauses is given a more compact $O(\mathrm{~km})$ representation and propagated in $O(\mathrm{~km})$ time (e.g., the e-clauses described in [5]). In the sequel we will assume that this optimization to the UP engine is used, so as to avoid an unnecessary $m^{2}$ factor in our complexity results.

In addition to the VDom clauses, the encoding contains additional clauses used to capture the constraint $C$. These constraint clauses utilize additional propositional variables $t_{1}, \ldots, t_{m}$, each one representing one of the $m$ different tuples over $\operatorname{dom}[\operatorname{scope}(C)]$ that satisfies $C$. Let $\tau_{i}$ be the satisfying tuple represented by the propositional variable $t_{i}$. Using the $t_{i}$ variables the clauses capturing $C$ are as follows:

1. For the variable $V \in \operatorname{scope}(C)$ and value $d \in \operatorname{dom}[V]$, let $\left\{s_{1}, \ldots, s_{i}\right\}$ be the subset of $\left\{t_{1}, \ldots, t_{m}\right\}$ such that the satisfying tuples represented by the $s_{i}$ are precisely the set of tuples containing $V=d$. That is, the $s_{i}$ represent all of the supports for $V=d$ in the constraint $C$. Then for each variable and value $V=d$ we have the clause $\left(s_{1}, \ldots, s_{i}, \neg A_{V=d}\right)$, which captures the condition that $V=d$ cannot be true if it has no support.

2. For each satisfying tuple of $C, \tau_{i}$, and assignment $V=d \in \tau_{i}$ we have the clause $\left(A_{V=d}, \neg t_{i}\right)$, which captures the condition that the tuple of assignments $\tau_{i}$ cannot hold if $V=d$ cannot be true.

When building this encoding, any value $d \in \operatorname{dom}[V]$ that is unsupported in $C$ will yield a unit clause $\neg A_{V=d}$ in item 1 . Thus for every value $d \in \operatorname{dom}[V]$ pruned by GAC, UP will force $\neg A_{V=d}$. More interestingly, in a dynamic context if $d$ is pruned (say by GAC on another constraint), then the clauses of item 2 will allow UP to negate all tuple variables $t_{i}$ such that $V=d \in \tau_{i}$. Then the clauses of item 1 will allow UP to delete any newly unsupported domain values. This propagation will continue until all unsupported domain values have been removed and GAC has been re-established.

It can also be observed that the size of this encoding (i.e., the sum of the lengths of the clauses) is linear in the size of the constraint's tabular representation: $O(m k)$ where $m$ is the number of satisfying tuples, and $k$ is the constraint's arity. In particular, $t_{i}$ appears in only $k$ clauses from item 1 (which bounds to total length of these clauses), and $k$ clauses from item 2 (which are all of length 2). Hence UP on this encoding will operate in time linear in the size of the constraint's tabular representation, i.e., the same time complexity as a generic GAC algorithm.

\section{UP for generic GAC revisited}

We now present an new method for achieving GAC on a generic constraint via UP. Our method does not introduce any new propositional variables (only assignment variables are used).

In the new encoding we have the VDom clauses encoding the multi-valued variables in scope $(C)$, as before. Then for each falsifying tuple of assignments $\tau=\left\langle V_{1}=\right.$ $\left.d_{1}, V_{2}=d_{2}, \ldots, V_{k}=d_{k}\right\rangle$ to the variables in scope $(C)$ we add the clause $\left(\neg A_{V_{1}=d_{1}}\right.$, $\ldots, \neg A_{V_{k}=d_{k}}$ ) which blocks this tuple of assignments. Call this set of clauses $\operatorname{direct}(C)$ [17]. Let the total set of clauses encoding $C$ be $T=\operatorname{VDom} \cup \operatorname{direct}(C)$. Now we replace $T$ with its set of prime-implicates. 
Definition 1 If $F$ is a CNF formula then the clause $c$ is a prime implicate of $F$ if $F \models c$ and for any $c^{\prime}$ that is a sub-clause of $c\left(c^{\prime} \subsetneq c\right)$, then $F \not \models c^{\prime}$.

Letting $P I(T)$ be the set of prime implicates of $T$ we have the following result.

Theorem 1. If the constraint $C$ is satisfied by some tuple of assignments, UP on the $C N F$ theory $P I(T)$ achieves GAC on $C$. That is, the assignment $V=d$ is GACinconsistent for $C$ iff UP on PI $(T)$ forces $\neg A_{V=d}$. If $C$ has no satisfying tuple, UP on $P I(T)$ will generate the empty clause (and GAC will generate an empty domain).

Proof: First we observe that the set of satisfying tuples of $C$ and the set of models of $T$ are in 1-1 correspondence. From any model of $T$ we must have one and only one assignment variable $A_{V=d}$ being true for each variable $V \in \operatorname{scope}(C)$. Furthermore, this set of true assignments do not correspond to any falsifying tuple, else they would falsify one of the clauses encoding $C$. Similarly, from a satisfying tuple for $C$ we can make the corresponding assignment variables of $T$ true, this will falsify all other assignment variables and thus satisfy all the clauses encoding $C$.

Hence, if GAC prunes $V=d, A_{V=d}$ cannot be true in any model of $T$, thus $T \models$ $\left(\neg A_{V=d}\right)$ and this unit clause must be part of $P I(T)$. On the other hand if UP on $P I(T)$ forces $\neg A_{V=d}$ then $A_{V=d}$ must be false in every model of $P I(T)$, hence false in every model of $T$ (since $P I(T)$ is logically equivalent to $T$ ), hence $V=d$ cannot be part of any satisfying tuple, and hence it will be pruned by GAC. Finally, if $C$ is unsatisfiable, $T$ will be UNSAT, and $P I(T)$ will contain only the empty clause.

Corollary 1 If after establishing GAC by running UP on $P I(T)$ we additionally prune the assignment $V=d$ (say by GAC on another constraint), then UP on $P I(T) \cup$ $\left(\neg A_{V=d}\right)$ will re-establish $G A C$.

This corollary can be seen to be true by observing that UP on $P T(T) \cup\left(\neg A_{V=d}\right)$ is the same as UP on $\left.P T(T)\right|_{\neg A_{V=d}}$ and that this is the set of prime implicates of $\left.T\right|_{\neg A_{V=d}}$.

Computing the set of prime implicates of a theory can be expensive; in the worst case it is exponential in the sum of the domain sizes of the variables in scope $(C)$. Nevertheless, we can make the following observations. First, the prime implicate encoding can be computed prior to search. Furthermore, once a constraint has been converted to its prime implicate encoding that encoding can be reused in any CSP containing the constraint. Second, some constraints might have a special structure that permit a more efficient computation of their prime implicates. And third even partial computation of some of the implicates of $T$ could allow UP to derive more consequences even if it fails to achieve GAC.

\section{Using a UP engine in a CSP solver}

The two encodings presented above facilitate achieving GAC on an arbitrary constraint, but the complexity remains exponential in the constraint's arity. In the next section we investigate encodings that exploit constraint specific structure to move from exponential to polynomial complexity. But before presenting those results we first discuss how an UP engine could be utilized in a CSP solver. 
The architecture would be as illustrated in the figure to the right. The UP engine operates on a set of clauses encoding some of the constraints $C_{1}, \ldots, C_{k}$. These clauses would also include the VDom clauses encoding variable domains. The other constraints of the problem, $D_{1}, \ldots, D_{i}$ are handled by a standard CSP propagator (e.g., a GAC propagator). Both UP and GAC communicate with each other and with an underlying solver. The communication required is simple. Whenever GAC (the solver) prunes a value $d$ from $\operatorname{dom}[V]$ (assigns $V=d$ ) it informs the UP engine that

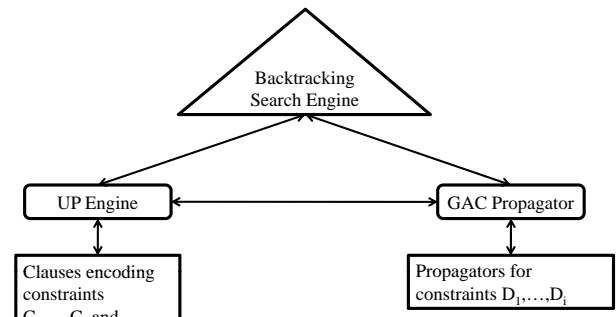
$\mathrm{C}_{1}, \mathrm{C}_{\mathrm{k}}$ and variable domains these constraints

the associated literal $\neg A_{V=d}\left(A_{V=d}\right)$ has become true. UP can then propagate this literal which might result in other variables $A_{X=a}$ being forced true or false. If $A_{X=a}$ becomes true, then the assignment $X=a$ is forced and all of $X$ 's other values have been pruned. The GAC engine can then propagate the fact that $X \neq d$ for all $d \neq a$. If $A_{X=a}$ becomes false, then the GAC engine can propagate the pruned value $X \neq a$. Either engine might be able derive a contradiction, at which point the search engine can backtrack. On backtrack the search engine will inform the GAC and UP engines to backtrack their state. For UP this is the simple process of unassigning all literals set to be true since the backtrack point.

Note that UP can also return a clausal reason for every assignment variable it forces. These clausal reasons can then be utilized by the search engine to perform nonchronological backtracking and clause learning. Since these clauses can contain both positive and negative instances of the assignment variables they are more general than standard nogoods (which contain only positive instances of assignment variables). As shown in [12] this added generality can yield a super-polynomial speedup in search. With the GAC engine, however, we have to modify each specialized propagator to allow it to produce an appropriate clausal reason for its pruned values. [12] gives some examples of how this can be accomplished.

\section{Constraint specific UP encodings}

GAC propagators are one of the fundamental enablers of GAC in CSP solvers. Propagators are constraint specific algorithms that exploit the special structure of a constraint so as to compute GAC in time polynomial in the constraint's arity.

Our main contribution is to demonstrate that constraint specific structure can also be exploited by a UP engine for a range of constraints. In particular, we present specialized clausal encodings for three different constraints, regular, among, and gen-sequence, such that UP on these encodings achieve GAC as efficiently as currently known propagators. 
There has been some previous work on exploiting structure via specialized CNF encodings of constraints, e.g., $[2,15,10,1]$. These works however are mostly aimed at obtaining better performing CNF encodings for use in a SAT solver. In particular, either UP on these encodings does not achieve GAC or when it does it is not as efficient as standard GAC propagators.

\subsection{Regular}

A regular constraint over a sequence of $k$ variables asserts that the sequence of values assigned to those variables falls into a specific regular language. By varying the language the regular constraint can be used to capture a number of other constraints (e.g., the stretch constraint) [13]. It is not always possible to achieve GAC on a regular constraint in time polynomial in its arity. In particular, the complexity of achieving GAC also depends on the complexity of the regular language being recognized. Nevertheless, UP on our clausal encoding can achieve the same complexity guarantees as the propagator given in [13].

More formally, each regular language $\mathcal{L}$ has an associated Deterministic Finite Automaton (DFA) $M$ that accepts a string iff that string is a member of $\mathcal{L}$. $M$ is defined by the tuple $\left\langle Q, \Sigma, \delta, q_{0}, F\right\rangle$, where $Q$ is a finite set of automaton states, $\Sigma$ is an input alphabet of symbols, $\delta$ is a transition function mapping state-input symbol pairs to new states $Q \times \Sigma \mapsto Q, q_{0}$ is the initial state, and $F$ is a set of accepting states. A DFA takes as input a string of characters. Each character $c \in \Sigma$ causes it to transition from its current state $q$ to the new state $\delta(q, c)$. The DFA starts off in the state $q_{0}$ and a string $S$ over the alphabet $\Sigma$ is said to be accepted by the DFA $M$ if $S$ causes $M$ to transition from $q_{0}$ to some accepting state in $F$.

Let $\mathcal{L}$ be a regular language over the alphabet of symbols $\bigcup_{i=1}^{k} \operatorname{dom}\left[V_{i}\right]$, and $M$ be a DFA that accepts $\mathcal{L}$. The regular constraint over the sequence of variables $\left\langle V_{1}, \ldots, V_{k}\right\rangle$ and the language $\mathcal{L}, \operatorname{regular}_{\mathcal{L}}\left(\left\langle V_{1}, \ldots, V_{k}\right\rangle\right)$ is satisfied by a sequence of assignments $\left\langle V_{1}=d_{1}, \ldots V_{k}=d_{k}\right\rangle$ iff the sequence of values $\left\langle d_{1}, \ldots, d_{k}\right\rangle$ is accepted by the DFA associated with $\mathcal{L}$.

Our clausal encoding of regular utilizes the insight of [13] that GAC can be achieved by looking for paths in a layered directed graph. The graph has $k$ layers each of which represents the possible states the DFA can be in after $s$ inputs $(0 \leq s \leq k)$. Any input string generates a path in this graph, and an accepted string must generate a path starting at $q_{0}$ and ending in some state of $F$. Our encoding captures the structure of this graph in such a way that UP can determine whether or not any particular value $d \in \operatorname{dom}\left[V_{i}\right]$ lies on an accepting path (is part of a satisfying tuple). The encoding contains the assignment variables $A_{V_{i}=d}\left(d \in \operatorname{dom}\left[V_{i}\right]\right)$ along with the VDom clauses encoding the variable domains. In addition it also contains the following variables and clauses:

1. For each step $s$ of $M$ 's processing, $0 \leq s \leq k$, and each state $q_{i} \in Q$, the state variable $q_{i}^{s}$. This variable is true if $M$ is in state $q_{i}$ after having processed $s$ input symbols.

2. For each transition $\left(q_{i}, d\right) \mapsto q_{j} \in \delta$ and each step $s, 1 \leq s \leq k$ such that $d \in$ $\operatorname{dom}\left[V_{s}\right]$, the transition variable $t_{q_{i}\langle d\rangle q_{j}}^{s}$. This variable is true if $M$ 's $s$-th input symbol is $d$ and on processing this symbol it transitions from state $q_{i}$ to $q_{j}$. 
3. For each transition variable the clauses $\left(\neg t_{q_{i}\langle d\rangle q_{j}}^{s}, q_{i}^{s-1}\right),\left(\neg t_{q_{i}\langle d\rangle q_{j}}^{s}, q_{j}^{s}\right)$, and $\left(\neg t_{q_{i}\langle d\rangle q_{j}}^{s}\right.$, $\left.A_{V_{s}=d}\right)$. These clauses capture the condition that if the transition $t_{q_{i}\langle d\rangle q_{j}}^{s}$ is true, $M$ must be in state $q_{i}$ at step $s-1, q_{j}$ at step $s$, and $V_{s}$ must be assigned the value $d$.

4. For each state variable $q_{i}^{s}$ let $\left\{t_{q_{i}\langle *\rangle *}^{s+1}\right\}$ be the set of transition variables representing transitions out of $q_{i}$ at step $s+1$ and $\left\{t_{*\langle *\rangle q_{i}}^{s}\right\}$ be the set of transition variables representing transitions into $q_{i}$ at step $s$. For each $q_{i}^{s}$ the encoding contains the clauses $\left(\left\{t_{q_{i}\langle *\rangle *}^{s+1}\right\}, \neg q_{i}^{S}\right)$ and $\left(\left\{t_{*\langle *\rangle q_{i}}^{s}\right\}, \neg q_{i}^{S}\right)$, capturing the condition that at least one incoming and one outgoing transition must be true for state $q_{i}$ to hold at step $s$. For step 0 we omit the clauses for incoming transitions, and for step $k$ we omit the clauses for outgoing transitions.

5. For each assignment variable $A_{V_{s}=d}$ let $\left\{t_{*\langle d\rangle *}^{s}\right\}$ be the set of transition variables representing transitions enabled by the assignment $V_{s}=d$ at step $s$. Then for each assignment variable the encoding contains the clause $\left(\left\{t_{*\langle d\rangle *}^{s}\right\}, \neg A_{V_{s}=d}\right)$ capturing the condition that at least one supporting transition must be true for this assignment to be possible.

6. For each state variable $q_{i}^{0}$ such that $i \neq 0$, the encoding contains the clause $\left(\neg q_{i}^{0}\right)$, capturing the condition that $M$ must start in step 0 in the initial state $q_{0}$. For each state variable $q_{i}^{k}$ such that $q_{i}$ is not an accepting state of $M$, the encoding contains the clause $\left(\neg q_{i}^{k}\right)$, capturing the condition that $M$ must finish in some accepting state.

Theorem 2. If the constraint regular ${ }_{\mathcal{L}}\left(\left\langle V_{1}, \ldots, V_{k}\right\rangle\right)$ is satisfied by some tuple of assignments, UP on the above encoding achieves GAC on the constraint in time $O(k m|Q|)$, where $k$ is the arity of the constraint, $m$ is the maximum sized domain, and $|Q|$ is the number of states of the DFA accepting $\mathcal{L}$. That is, the assignment $V_{i}=d$ is $G A C$-inconsistent for regular $\mathcal{L}_{\mathcal{L}}\left(\left\langle V_{1}, \ldots, V_{k}\right\rangle\right)$ iff UP on this encoding forces $\neg A_{V_{i}=d}$. If regular $_{\mathcal{L}}\left(\left\langle V_{1}, \ldots, V_{k}\right\rangle\right)$ has no satisfying assignment $U P$ will generate the empty clause (GAC will generate an empty domain).

Proof: First we address the size of the encoding. There are $|Q| k$ state variables (where $k$ is the arity of the constraint and $|Q|$ is the number of states of $M$ ), and if $m$ is the maximal sized domain of any of the variables $V_{i}$, at most $k m|Q|$ transition variables. In particular, $M$ is deterministic, hence for each transition variable $t_{q_{i}\langle d\rangle q_{j}}^{s}$ if any two of the three parameters $q_{i}, d$, or $q_{j}$ are fixed the last parameter can only have a single value ( $s$ ranges from 1 to $k$ ).

Associated with each transition variable are 3 clauses from item 3 for a total size of $O(k m|Q|)$. Associated with each of the $k|Q|$ state variables is a clause of length $\left|\left\{t_{q_{i}\langle *\rangle *}^{s+1}\right\}\right|+1$ and a clause of length $\left|\left\{t_{*\langle *\rangle q_{i}}^{s}\right\}\right|+1$, from item 4 . These clauses are at most $m+1$ in length (given the input symbol the other (input or output) state is determined): again a total size of $O(\mathrm{~km}|Q|)$. Finally, associated with each of the $O(\mathrm{~km})$ assignment variables is a single clause of length $\left|\left\{t_{*\langle d\rangle *}^{s}\right\}\right|+1$. These clauses can be most length $|Q|+1$ (fixing the input or output state determines the other state): once again a total size of $O(k m|Q|)$. Hence the total size of the clausal encoding is $O(k m|Q|)$ which gives the space as well as the time complexity of UP (UP runs in time linear in the total 
size of the encoding). ${ }^{2}$ This is the same time and space complexity of the propagator given in [13].

Now we prove that UP achieves GAC assuming that the constraint is satisfied by some tuple of assignments. First, if $V_{i}=d_{i}$ is not pruned by GAC then UP cannot force $\neg A_{V_{i}=d_{i}}$. If $V_{i}=d_{i}$ is not pruned by GAC it must have a supporting tuple, $\left\langle V_{1}=d_{1}, \ldots, V_{i}=d_{i}, \ldots, V_{k}=d_{k}\right\rangle$. This sequence of inputs to $M$ will cause $M$ to transition through a sequence of states $\left\langle q_{\pi(0)}, q_{\pi(1)}, \ldots, q_{\pi(k)}\right\rangle$ starting at the initial state $q_{\pi(0)}=q_{0}$ and ending at an accepting state $q_{\pi(k)} \in F$. Setting all of the corresponding assignment variables $A_{V_{i}=d_{i}}$, state variables $q_{\pi(s)}^{s}$, and transition variables $t_{q_{\pi(s-1)}^{s}\left\langle d_{s}\right\rangle q_{\pi(s)}}$ to be true, and and all other variables to be false, we observe that all clauses become satisfied. Hence $A_{V_{i}=d_{i}}$ is part of a satisfying truth assignment and since UP is sound it cannot force $\neg A_{V_{i}=d_{i}}$. We conclude by contraposition that if UP forces $\neg A_{V_{i}=d_{i}}$ then GAC must prune $V_{i}=d_{i}$

Second, if UP does not force $\neg A_{V_{i}=d_{i}}$ then GAC will not prune $V_{i}=d_{i}$, and by contraposition we have that if GAC prunes $V_{i}=d_{i}$ then UP must force $\neg A_{V_{i}=d_{i}}$. Given that UP has been run to completion and $\neg A_{V_{i}=d_{i}}$ has not been forced, then by the clauses of item 5 there must exist some transition variable $t_{q_{h}\left\langle d_{i}\right\rangle q_{j}}^{i}$ that also has not be falsified by UP. By the clauses of item 2, it must also be the case that the state variables $q_{h}^{i-1}$ and $q_{j}^{i}$ have not been falsified. By the clauses of item 4 there must be corresponding transition variables $t_{q_{g}\left\langle d_{i-1}\right\rangle q_{h}}^{i-1}$ incoming to $q_{h}^{i-1}$ and $t_{q_{j}\left\langle d_{i+1}\right\rangle q_{k}}^{i+1}$ outcoming from $q_{j}^{i}$, neither of which have been falsified. By the clauses of item 2 the assignment variables $A_{V_{i-1}=d_{i-1}}$ and $A_{V_{i+1}=d_{i+1}}$ cannot be falsified. Continuing this way we arrive at a input sequence that includes $V_{i}=d_{i}$ and that causes $M$ to transition from $q_{0}$ to an accepting state. That is, $V_{i}=d_{i}$ has a supporting tuple, and GAC will not prune it.

Finally, if $\operatorname{regular}_{\mathcal{L}}\left(\left\langle V_{1}, \ldots, V_{k}\right\rangle\right)$ has no satisfying assignment then no value $d \in$ $\operatorname{dom}\left[V_{i}\right]$ is supported (for any variable $V_{i}$ ). By the above UP will force $\neg A_{V_{i}=d}$ for every $d \in \operatorname{dom}\left[V_{i}\right]$ thus making the VDom clause $\left(A_{V_{i}=d_{1}}, \ldots, A_{V_{i}=d_{m}}\right)$ empty.

Corollary 2 If after initial GAC we additionally prune the assignment $V_{i}=d_{i}$, then adding the unit clause $\neg A_{V_{i}=d_{i}}$ and again performing UP re-establishes GAC.

That is, we can incrementally maintain GAC on $\operatorname{regular}_{\mathcal{L}}\left(\left\langle V_{1}, \ldots, V_{k}\right\rangle\right)$ by simply falsifying the assignment variables for every pruned assignment and then redoing UP. As noted in the introduction UP is inherently incremental, so no changes need to be made to achieve an incremental propagator. This corollary can be proved by observing that the proof of the theorem continues to apply even if some of the assignment variables have initially been set to be false.

In [14] a CNF encoding for the grammar constraint has been developed. That encoding is based on the grammar rules view of languages, and uses CNF to encode a dynamic programming parsing algorithm. UP on the encoding is able to achieve GAC for context free grammar constraints. Since regular languages are a subset of context free languages, this encoding supplies an alternate way of using UP to achieve GAC on regular. However, as demonstrated below our encoding for regular can be extended to provide an encoding for gen-sequence whereas the encoding of [14] does not provide

\footnotetext{
${ }^{2}$ As discussed Section 2.1 we are exploiting the fact that the $\mathrm{km}^{2}$ VDom clauses can be represented and propagated in $O(\mathrm{~km})$ space and time.
} 
an immediate solution for gen-sequence. Furthermore, the argument that UP might be a useful general way of achieving GAC is not put forward in that paper.

\subsection{Among}

An among constraint over a sequence of $k$ variables, $\left\langle V_{1}, \ldots, V_{k}\right\rangle$ asserts that at least min and at most max of these variables can take on a value from a specific set $S$. More formally, among $\left(\left\langle V_{1}, \ldots, V_{k}\right\rangle, S, \min , \max \right)$ is satisfied by a tuple of assignments $\left\langle d_{1}, \ldots, d_{k}\right\rangle$ iff $\min \leq\left|\left\langle d_{1}, \ldots, d_{k}\right\rangle \cap S\right| \leq \max$.

To simplify our notation we can consider among $\left(\left\langle V_{1}, \ldots, V_{k}\right\rangle,\{1\}\right.$, min, $\left.\max \right)$ in which all of the variables in $\left\langle V_{1}, \ldots, V_{k}\right\rangle$ have domain $\{0,1\}$ and $S$ is $\{1\}$. Any among constraint among $\left(\left\langle X_{1}, \ldots, X_{k}\right\rangle, S^{\prime}, \min , \max \right)$ can be reduced to this case by introducing the $\left\langle V_{1}, \ldots, V_{k}\right\rangle$ as new variables and imposing a constraint between $X_{i}$ and $V_{i}$ $(1 \leq i \leq k)$ such that $V_{i}=1$ iff $X_{i}$ has a value in $S^{\prime}$. In fact, although we omit the details here, the constraint between the original $X_{i}$ variables and the $V_{i}$ variables can be captured with a CNF encoding and enforced with the same UP engine used to enforce among.

We can specify a CNF encoding for among by constructing a DFA that has max states, $q_{0}, \ldots, q_{\max }$. The states $q_{\min }, \ldots q_{\max }$ are all accepting states. The input alphabet is the two conditions $V_{i}$ and $\neg V_{i}$ (the $V_{i}$ are binary values thus they can be treated as propositions with $V_{i} \equiv V_{i}=1$ and $\neg V_{i} \equiv V_{i}=0$ ). The transition function is simply defined by the condition that an $V_{i}$ input causes a transition from $q_{j}$ to $q_{j+1}$, while a $\neg V_{i}$ input causes a transition from $q_{j}$ back to $q_{j}$. In other words, the states of the DFA simply keep track of the number of variables taking values in $S$ and accepts iff the total number over all $k$ variables lies in the range [min, max]. Thus the among constraint can be encoded as a CNF on which UP achieves GAC by using the previously specified encoding for this DFA.

Encoding among as a CNF is not particularly practical, as among has a very simple propagator that is more efficient than UP on this CNF encoding. The real use of the CNF encoding of among comes from applying it to conjunctions of among constraints. It can also be noted that $[1,15]$ both provide CNF encodings for the Boolean Cardinality Constraint which is equivalent to the above among constraint. Their encodings allow UP to achieve GAC, but as with the above encoding, their encodings are not as efficient as the standard propagator.

\subsection{Generalized Sequence Constraint}

A gen-sequence constraint over a sequence of variables $\left\langle V_{1}, \ldots, V_{k}\right\rangle$ is a conjunction of among constraints. However, it is not an arbitrary collection of among constraints. In particular, all of the among constraints are over sub-sequences of the same global sequence $\left\langle V_{1}, \ldots, V_{k}\right\rangle$. Furthermore, the among constraint all count membership in a fixed subset of domain values $S .{ }^{3}$ More formally,

$$
\begin{gathered}
\text { gen-sequence }\left(\left\langle V_{1}, \ldots, V_{k}\right\rangle, S,\left\langle\sigma_{1}, \ldots, \sigma_{m}\right\rangle,\left\langle\min _{1}, \ldots, \min _{m}\right\rangle,\left\langle\max _{1}, \ldots, \max _{m}\right\rangle\right) \\
\equiv \bigwedge_{j=1}^{m} \text { among }_{j}\left(\sigma_{j}, S, \min _{j}, \max _{j}\right),
\end{gathered}
$$

\footnotetext{
${ }^{3}$ The algorithm provided in [16] also requires that the set $S$ be fixed over all among constraints.
} 
where each $\sigma_{j}$ is a sub-sequence of $\left\langle V_{1}, \ldots, V_{k}\right\rangle$.

We present an encoding on which the failed literal test achieves GAC. That is, with this encoding, each value of each variable can be tested to determined if it is supported by doing a single run of UP. It is particularly important that modern implementations of UP are decremental almost without cost. Thus all that needs to be done between testing successive variable values is to undo the truth assignments forced by the previous run of UP. Using the failed literal test to achieve GAC on this constraint achieves the same time complexity guarantee as the propagator given in [16].

Since the set $S$ is fixed across all of the among constraints, we can apply the same transformation used in the previous section to convert them to among constraints over variables with domain $\{0,1\}$ and $S=\{1\}$. With this simplifying transformation our CNF encoding for gen-sequence consists of the encoding generated by a simple DFA along with some additional clauses. In particular, to obtain a CNF encoding for gen-sequence $\left(\left\langle V_{1}, \ldots, V_{k}\right\rangle,\{1\},\left\langle\sigma_{1}, \ldots, \sigma_{m}\right\rangle,\left\langle\min _{1}, \ldots, \min _{m}\right\rangle,\left\langle\max _{1}, \ldots, \max _{m}\right\rangle\right)$ (abbreviated as gen-sequence $\left(\left\langle V_{1}, \ldots, V_{k}\right\rangle\right)$ ) where $S=\{1\}$ and the variables are all have domain $\{0,1\}$, we first generate the $\mathrm{CNF}$ encoding for $\operatorname{among}\left(\left\langle V_{1}, \ldots, V_{k}\right\rangle, S, 0, k\right)$.

This encoding captures a DFA that simply keeps track of the number of variables in $\left\langle V_{1}, \ldots, V_{k}\right\rangle$ that lie in $S$ : all states are accepting. In the CNF encoding each step $s$ $(0<s \leq k)$ contains $s$ state variables $q_{i}^{s}(0 \leq i \leq s)$ indicating that $i$ of the first $s$ variables $V_{1}, \ldots, V_{s}$ took a value in $S$. The transition variables $t_{q_{i}\left\langle V_{s}\right\rangle q_{i+1}}^{s}$ and $t_{q_{i}\left\langle\neg V_{s}\right\rangle q_{i}}^{s}$ capture the transitions $q_{i} \rightarrow q_{i+1}$ made when $V_{s}$ takes a value in $S$ and $q_{i} \rightarrow q_{i}$ made when $V_{s}$ takes a value not in $S$.

In addition to the clauses encoding this "base" DFA, for every constraint among $_{j}\left(\sigma_{j}\right.$, $\left.S, \min _{j}, \max _{j}\right)$ in the gen-sequence we have the following clauses.

1. Let $\sigma_{j}$ be the subsequence of variables $\left\langle V_{g}, \ldots, V_{h}\right\rangle$, for every state variable at step $g-1, q_{i}^{g-1}(0 \leq i \leq k)$ we have the clause $\left(q_{i+\min _{j}}^{h}, \ldots, q_{i+\max _{j}}^{h}, \neg q_{i}^{g-1}\right)$ encoding the condition that if the count by time we have reached step $h$ does not lie in the range $\left[i+\min _{j}, i+\max _{j}\right]$ then we cannot have started with the count at $i$ at step $g-1$.

2. The clause $\left(q_{i-\max _{j}}^{g-1}, \ldots, q_{i-\min _{j}}^{g-1}, \neg q_{i}^{h}\right)$ encoding the condition that if the count by time we reach step $h$ is $i$ then we must have started off in the range $\left[i-\max _{j}, i-\right.$ $\min _{j}$ ] at step $g-1$.

Theorem 3. If the constraint gen-sequence $\left(\left\langle V_{1}, \ldots, V_{k}\right\rangle\right)$ is satisfied by some tuple of assignments, the failed literal test on the above encoding achieves GAC on the constraint in time $O\left(m k^{3}\right)$, where $k$ is the arity of the constraint, and $m$ is the number of among constraints in the gen-sequence constraint. That is, the assignment $V_{i}$ (i.e., $\left.V_{i}=1\right)$ is GAC-inconsistent iff the failed literal test on $V_{i}$ yields a contradiction. Similarly for the assignment $\neg V_{i}$ (i.e., $V_{i}=0$ ). If gen-sequence $\left(\left\langle V_{1}, \ldots, V_{k}\right\rangle\right)$ has no satisfying assignment then UP (without use of the failed literal test) will generate the empty clause (GAC would generate an empty domain). ${ }^{4}$

\footnotetext{
${ }^{4}$ Note that in [16] the claim is made that their propagator runs in time $O\left(k^{3}\right)$. However, a close look at their algorithm demonstrates that it in fact requires $O\left(m k^{3}\right)$. In particular, each time a PUSHUP operation (at most $O\left(k^{2}\right)$ ) in their CHECKCONSISTENCY algorithm is performed
} 
Proof: We show that $V_{i}$ is GAC inconsistent iff the failed literal test on $\neg V_{i}$ yields a contradiction. The argument for $\neg V_{i}$ is similar.

Consider the relevant parts of the CNF encoding of gen-sequence. These are the clauses encoding the base DFA that counts the number of variables taking values in $S$, along with the clauses of item 1 and 2 above capturing the relationship between the initial and final counts for each among constraint.

If GAC does not prune $V_{i}$ then there must be some supporting tuple of values containing it $\left\langle V_{1}, \ldots, V_{k}\right\rangle$. We can then generate a path through the base DFA from this input sequence of $V_{j}$ values. As with the proof of Theorem 2 this sequence can be used to assign true to the state, and transition lying along this accepting path, with all other variables assigned false. It can then be observed that all clauses of the DFA will be satisfied, and further since the sequence also satisfies all $m$ among constraints so will all clauses for the $m$ among constraints (items 1 and 2 above). Thus UP cannot not force $\neg V_{i}$ since $V_{i}$ is part of a satisfying assignment. Furthermore, the failed literal test on $V_{i}$ cannot yield a contradiction. By contraposition we conclude that if the failed literal test yields a contradiction then GAC will prune the value.

To prove the other direction consider what happens after UP (but not failed literal) has been run. There will be some set of unfalsified state variables $Q_{s}$ at each step $s$. If any of these sets, say $Q_{i}$ is empty, UP must have produced an empty clause. In particular, the clauses of item 3 of the DFA encoding (specifying that a transition cannot be true if its final state is false) will allow UP to falsify all incoming transitions variables into states of step $s$, and this in turn will allow UP to falsify both "alphabet symbols" $V_{i}$ and $\neg V_{i}$ using the clauses of item 5 of the DFA. This will give rise to a contradiction and an empty clause. By the previous paragraph this means that the constraint has no satisfying tuple, as each satisfying tuple yields a model of the clauses.

Say that none of these sets $Q_{s}$ is empty, let $\left\langle q_{0}, q_{\pi(1)}, \ldots, q_{\pi(k)}\right\rangle$ be the sequence of states such that $\pi(i)$ is the minimal indexed state variable of $Q_{i}$. This sequence of states corresponds to the minimal unfalsified counts in the DFA after UP. We claim that this sequence of states corresponds to a satisfying tuple for gen-sequence. First, we have that either $\pi(i+1)=\pi(i)$ or $\pi(i+1)=\pi(i)+1$, that is these counts can increase by zero or one at each step. If $\pi(i+1)<\pi(i)$, then the only transitions into $q_{\pi(i+1)}^{i+1}$ are from $q_{j}^{i}$ with $j<\pi(i)$. However, all such state variables have been falsified as $q_{\pi(i)}^{i}$ is the minimal indexed state variable at step $i$, hence the clauses of item 3 of the DFA would have falsified all transitions variables coming into $q_{\pi(i-1)}^{i+1}$, and the clauses of item 4 of the DFA would falsify $q_{\pi(i+1)}^{i+1}$ : a contradiction. Similarly, if $\pi(i+1)>\pi(i)+1$ then all outgoing transitions from $q_{\pi(i)}^{i}$ would be falsified and so would $q_{\pi(i)}^{i}$. Second, if $\pi(i+1)=\pi(i)$ the variable $\neg V_{i}$ cannot be false and if $\pi(i+1)=\pi(i)+1$ the variable $V_{i}$ cannot be false: if these variables were false then one or both of $q_{\pi(i)}^{i}$ and $q_{\pi(i+1)}^{i+1}$ would fail to have any incoming or outgoing transitions and would thus be falsified by UP. Finally, this sequence must satisfy each among constraint because of the clauses from each of these constraints. Consider the clauses $\left(q_{\pi\left(g+\min _{j}\right)}^{h}, \ldots, q_{\pi\left(g+\max _{j}\right)}^{h}, \neg q_{\pi(g-1)}^{g-1}\right)$

it could require first checking all $m$ among constraints to see if any are violated. This yields an $O\left(m k^{2}\right)$ complexity for CHECKCONSISTENCY and an overall $O\left(m k^{3}\right)$ complexity for achieving GAC. 
and $\left(q_{\pi\left(h-\max _{j}\right)}^{g-1}, \ldots, q_{\pi\left(h-\min _{j}\right)}^{g-1}, \neg q_{\pi(h)}^{h}\right)$ from among constraint $j$. Since $q_{\pi(g-1)}^{g-1}$ is the minimal indexed state variable at step $g$ and $q_{\pi(h)}^{h}$ is the minimal indexed state variable at step $h$, we can see that if $\pi(g)-\pi(h)$ lies outside of the range $\left[\min _{j}, \max _{j}\right]$ then one of $q_{\pi(g-1)}^{g-1}$ or $q_{\pi(h)}^{h}$ will be falsified by UP from these clauses. For example, if $\pi(g)-\pi(h)>\max _{j}$ then all of the step $h$ state literals in $\left(q_{\pi\left(g+\min _{j}\right)}^{h}, \ldots, q_{\pi\left(g+\max _{j}\right)}^{h}\right.$, $\left.\neg q_{\pi(g-1)}^{g-1}\right)$ would be falsified and thus $q_{\pi(g-1)}^{g-1}$ would also be falsified by UP. Hence, these minimal states and the corresponding transitions and $V_{j}$ variables between them define a satisfying tuple for gen-sequence. We also see that if the constraint has no satisfying tuple, at least one of the sets $Q_{s}$ must be empty and by the previous paragraph UP yields the empty clause. This proves the last claim of the theorem.

If we apply the failed literal test to $V_{i}$, this will force $V_{i}$ and thus if there is no contradiction the minimal path after UP must contain $V_{i}$ in the satisfying tuple it defines. Thus $V_{i}$ is supported, and so GAC will not prune this assignment. By contraposition, if GAC prunes $V_{i}$ then the failed literal test must generate a contradiction.

Turning now to the complexity, note that the encoding for the base DFA is of size (total length of clauses) $O\left(k^{2}\right)$; in particular $|Q|=k$ and $m=2$ since the base DFA only has to symbols $V_{j}$ and $\neg V_{j}$ in its alphabet. The clauses encoding the among constraints total $m$ (the number of among constrains) times $O\left(k^{2}\right)$; each among constraint generates $k$ clauses from item 1 and $k$ clauses of item 2 , each of these clauses is of length $O(k)$. So in total we have a CNF theory of total size $O\left(m k^{2}\right)$. At most we have to do $O(k)$ failed literal tests each of which runs in time $O\left(k^{2}\right)$. This gives us a total complexity for achieving GAC of $O\left(m k^{3}\right)$ in the worst case.

Some additional comments can be made about the practicality of this approach to GAC on gen-sequence. First, the failed literal test can be quite efficient. In particular, no work needs to be done to reset the "data structures" (i.e., the clauses) between successive failed literal tests. Second, UP on this encoding achieves incremental GAC, it is not difficult to see that if we prune values from the domains of the variables $V_{i}$ so as to force the value of $V_{i}$, UP can incrementally re-establish GAC. Third, the initial UP and each successive failed literal test yields a supporting tuple. If $V_{i}$ or $\neg V_{i}$ appears in any of these tuples, we need not do a failed literal test on it: it is already supported. Furthermore, by analogous reasoning it is not hard to see that the sequence of maximum indexed state variables in the sets $Q_{s}$ also forms a satisfying tuple. Thus the initial UP and each subsequent failed literal test can yield up to two satisfying tuples, each of which can be used to avoid testing some as yet untested $V_{i}$ literals.

\section{Conclusions}

In this paper we have shown that GAC can be efficiently achieved by using the approach of converting a constraint to a CNF and then employing a UP engine to do propagation in that CNF. As discussed in the introduction this approach has a number of advantages, including the fact that it is immediately incremental and decremental. We have also shown that special structure in a constraint can in some cases be exploited so that UP can achieve GAC with the same complexity as a constraint specific propagation algorithm. 
A number of questions remain open. First, there is the empirical question of how this approach performs in practice. On that front we are quite optimistic given the highly tuned nature of publically available UP engines. Second, there is the question of just how general is this approach; can it be applied to other well known constraints? One thing to note with respect to this question is that regular is already quite a general constraint. Nevertheless, further research along this line is definitely required. It should be noted that the CNF encodings presented here exploits structure that had already been uncovered and exploited in previous propagators. So it is feasible that other propagation algorithms could similarly exploited.

\section{References}

1. Olivier Bailleux and Yacine Boufkhad. Efficient cnf encoding of boolean cardinality constraints. In Proc. of CP2003, pages 108-122, 2003.

2. Olivier Bailleux and Yacine Boufkhad. Full cnf encoding: The counting constraints case. In Proc. of SAT-2004, 2004.

3. H. Bennaceur. A comparison between sat and csp techniques. Constraints, 9:123-138, 2004.

4. C. Bessière and J.C. Régin. Arc consistency for general constraint networks: Preliminary results. In Proc. IJCAI, pages 398-404, 1997.

5. Mark Chavira and Adnan Darwiche. Compiling bayesian networks with local structure. In Proc. IJCAI-2005, pages 1306-1312, 2005.

6. Y. Dimopoulos and K Stergiou. Propagation in csp and sat. In Proc. of CP2006, volume 4204 of LNCS, pages 137-151. Springer-Verlag, 2006.

7. Niklas En and Niklas Srensson. Minisat solver. http://www. cs. chalmers.se/Cs/ Research/FormalMethods/MiniSat/Minisat.html.

8. J. W. Freeman. Improvements to Propositional Satisfiability Search Algorithms. $\mathrm{PhD}$ thesis, University of Pennsylvania, 1995.

9. Ian Gent. Arc consistency in sat. In ECAI, pages 121-125, 2002.

10. Ian Gent and Peter Nightingale. A new encoding of all-different into SAT. In Proc. 3rd International Workshop on Modelling and Reformulating Constraint Satisfaction Problems, pages 95-110. 2004.

11. E. Hebrard, C. Bessière, and T. Walsh. Local consistencies in sat. In Proc. of SAT-2003, pages 400-407, 2003.

12. G. Katsirelos and F. Bacchus. Generalized nogoods in csps. In Proc. of AAAI, pages 390396, 2005.

13. G. Pesant. A regular language membership constraint for finite sequences of variables. In Proc. of CP-2004, 2004.

14. Claude-Guy Quimper and Toby Walsh. Decomposing global grammar constraints. In Proc. of CP2007, 2007.

15. Carsten Sinz. Towards an optimal cnf encoding of boolean cardinality constraints. In Proc. of CP2005, pages 827-831, 2005.

16. W.J. van Hoeve, G. Pesant, L.M. Rousseau, and A. Sabharwal. Revisiting the Sequence Constraint. In Proc. of CP-2006, 2006.

17. Toby Walsh. Sat v csp. In Proc. of CP2000, number 1894 in Lecture Notes in Computer Science, pages 441-456. Springer-Verlag, New York, 2000. 